\title{
Qualidade de vida de pacientes com migrânea relacionada ao período menstrual submetidos à terapia auricular
}

\section{Life quality of patients with migraine related to menstrual period undergoing auricular therapy}

\author{
Vivian Viani Picanço ${ }^{1}$; Karen Andréa Comparin²; Feng Hsiu Hsieh³ ${ }^{3}$ Deborah \\ Sandra Leal Guimarães Schneider ${ }^{4}$; Celeide Pinto Aguiar Peres ${ }^{5}$; \\ Joseane Rodrigues da Silva ${ }^{6}$
}

\section{Resumo}

A migrânea é uma doença crônica caracterizada por crises intermitentes de cefaléia e sintomas associados, e constitui uma afecção que acomete em torno de 12 a $15 \%$ da população em geral, em sua maioria mulheres. A qualidade de vida desses indivíduos fica comprometida, gerando um grande impacto físico, mental e social para os migranosos. Um dos métodos de tratamento que se mostra eficaz para aliviar os sintomas da migrânea menstrual é a terapia auricular ou auriculoterapia. O objetivo dessa pesquisa foi avaliar a qualidade de vida de pacientes com migrânea relacionada ao período menstrual que foram submetidos à terapia auricular. Para tanto, foram realizadas sessões de auriculoterapia em pacientes previamente selecionados e aplicado o questionário de qualidade de vida SF-36, em três momentos, antes da primeira sessão, sessenta dias após o início do tratamento e uma terceira avaliação noventa dias após o início do tratamento. Os resultados demonstraram que dos oitos indivíduos estudados, sete tiveram uma melhora geral da sua qualidade de vida. Conclui-se, assim, que o tratamento com a terapia auricular foi eficaz para a melhora da qualidade de vida de mulheres com migrânea relacionada ao período menstrual.

Palavras-chave: Migrânea. Período menstrual. Terapia auricular. Qualidade de vida.

\begin{abstract}
Migraine is a chronic disease characterized by intermittent attacks of headache and associated symptoms. It is a disease that affects around 12 to $15 \%$ of the general population, most of them being women. The quality of life of these individuals is compromised, causing a major impact on physical, mental and social for those who suffer from migraine. One method of treatment that seems to be effective in relieving the symptoms of menstrual migraine is auricular therapy. The purpose of this study was to evaluate the quality of life of patients with migraine related to the menstrual period who underwent auricular therapy. To this end, sessions were held in patients previously selected and implemented the survey of quality of life SF-36 on three different occasions. Before the first session, sixty days after the start of treatment and a third evaluation, ninety days after the beginning of treatment. The results showed that from eight individuals, seven had a general improvement in their quality of life. It follows
\end{abstract}

Unioeste, Acadêmica do $5^{\circ}$ ano de Fisioterapia da Universidade Estadual do Oeste do Paraná (Unioeste). Contato principal para correspondência. vivian.viani@gmail.com.

Unioeste, Fisioterapeuta Mestre; docente do curso de Fisioterapia da Unioeste. karencomparin@gmail.com.

Fisioterapeuta especializanda em acupuntura pelo Colégio Brasileiro de Acupuntura (CBA). feng_hs27@yahoo.com.br.

Estatística Mestre; docente da Unioeste. deborahschneider@uol.com.br.

Fisioterapeuta Doutora; docente do curso Fisioterapia da Unioeste. cperes@certto.com.br.

Fisioterapeuta Mestre; docente do curso de Fisioterapia da Unioeste.joseane_rs@yahoo.com.br 
that the treatment with auricular therapy was effective for improving quality of life of women with migraine related to menstruation.

Keywords: Migraine. Menstrual period. Auricular therapy. Quality of life.

\section{Introdução}

Migrânea é um termo de origem greco-latina popularmente conhecida como enxaqueca, e classificada como uma doença crônica com características de crises intermitentes de cefaléia e sintomas associados. A maioria das pessoas acometidas são mulheres (18-20\%), seguido de crianças $(8 \%)$ e homens $(6 \%)$, e na população em geral aparece em 12 a 15\% (KRYMCHANTOWSKY et al., 2004).

A crise de migrânea é caracterizada pelo aparecimento de dores de cabeça recorrentes que duram de 4 a 72 horas, uni ou bilateralmente, em geral com caráter pulsátil. A intensidade varia de moderada a forte, precedida em minutos ou não de sintomas neurológicos focais, denominados de aura, tais como escotomas, alterações visuais, alterações motoras e/ou na fala. Ela pode vir acompanhada de náuseas, vômito, fotofobia, osmofobia e/ou fonofobia (SOUZA, 2000; ZUKERMAN, 2000).

Segundo Bigal et al (2000), a enxaqueca prejudica mais a qualidade de vida $(\mathrm{QV})$ que a osteoartrite, diabetes, hipertensão e lombalgia e é tão incapacitante quanto a depressão. E são vários os motivos que comprometem a $\mathrm{QV}$ nesses indivíduos: maior incidência de dores no corpo, limitação física e consideram-se com menor saúde mental; comprometimento de suas vidas profissionais devido ao maior número de faltas e diminuição das atividades laborais secundárias à dor ou aos sintomas associados; o nível de ganho monetário dos enxaquecosos é menor que o da população geral; e a vida social é prejudicada. Todos esses fatores representam um grande impacto físico, mental e social para os migranosos, bem como implicam em altos custos para a sociedade.

Outros estudos ressaltam os efeitos negativos da migrânea na QV desses indivíduos acometidos, além do comprometimento da vida profissional, as atividades familiares e de lazer também são muito prejudicadas pelas crises de dores. Outra questão é que muitos migranosos são menosprezados e discriminados por pessoas que não sofrem dessas dores, e acabam minimizando os sintomas de quem os têm (GIONA, 2003).

A Sociedade Brasileira de Cefaléia relata que a mulher tem uma maior preponderância de apresentar enxaqueca quando comparado com os homens em uma proporção de 1: 2-3. As razões para este acontecimento estão relacionadas com os hormônios femininos, sendo assim, uma das cefaléias mais importantes na mulher é a migrânea menstrual, ou seja, enxaqueca que ocorre antes, durante ou imediatamente após a menstruação (SOCIEDADE BRASILEIRA DE CEFALÉIA, 2009).

Entre os recursos para se tratar desta afecção, a acupuntura, técnica milenar chinesa, tem sua comprovada eficácia na prevenção e tratamento de diversas doenças, principalmente aquelas com sintomas dolorosos (ARAÚJO; ZAMPAR; PINTO, 2006). A palavra acupuntura originou-se do latim, sendo que acus significa agulha e punctura significa puncionar (GONZALES, 2004). Essa técnica faz parte de um conjunto de conhecimentos teóricoempíricos, da medicina tradicional chinesa (MTC) e tem como objetivo o tratamento e a cura das enfermidades pela aplicação de estímulos através da pele, com a inserção de agulhas em pontos específicos do corpo (SCOGNAMILLO-SZABÓ; BECHARA, 2001).

A terapia auricular ou auriculoterapia é um ramo da acupuntura, que utiliza o pavilhão auricular para o tratamento de enfermidades físicas e mentais. Acredita-se que no pavilhão auricular existem 
pontos de reflexo que correspondem a todos os órgãos e funções do corpo, e ao serem estimulados, o cérebro recebe um impulso que desencadeia uma série de fenômenos físicos relacionados com a área do corpo, produzindo a cura (SOUZA, 2001).

Segundo Lowe (1973 apud SOUZA, 2001) em sua obra "Introduction to acupunture anesthesia", a orelha possui inervação abundante, obtida através dos nervos trigêmio, facial, vago, auriculares maiores e occipitais maiores e menores. Essas inervações, quando estimuladas, sensibilizam regiões do tronco cerebral, córtex e cerebelo. Cada ponto auricular tem relação direta com um ponto cerebral o qual, por sua vez, está ligado pela rede do sistema nervoso, a determinado órgão ou região do corpo comandando suas funções.

A migrânea menstrual atinge altos índices de mulheres, gerando incapacidades. Suas vidas ficam comprometidas em vários aspectos, conforme descreve a literatura, influenciando na qualidade de vida. Uma das formas de tratamento que vem amenizando muito as enxaquecas menstruais é a terapia auricular, método simples, econômico, prático e destituído de efeitos colaterais. Várias pesquisas se detêm exclusivamente em verificar a eficácia das técnicas de tratamento, mas não avaliam se o método influencia na qualidade de vida dos pacientes. Melhorar a qualidade de vida significa intervir positivamente nas condições de vida, sejam elas físicas, sociais, emocionais e mentais.

Sendo assim, diante do exposto, este estudo teve como objetivo avaliar a qualidade de vida de pacientes com migrânea relacionada ao período menstrual que foram submetidos à terapia auricular.

\section{Material e métodos}

A presente pesquisa caracteriza-se como quantitativa e utilizou como base de tratamento a terapia auricular, por meio de pontos fixos de acupuntura indicados para migrânea menstrual. Foi desenvolvida na Clínica de Fisioterapia da Universidade Estadual do Oeste do Paraná (Unioeste), campus de Cascavel, na qual foram selecionadas para o estudo mulheres com sintomatologia de migrânea menstrual.

Os critérios de inclusão foram os seguintes: mulheres com idade entre 18 e 40 anos que apresentavam migrânea relacionada à menstruação. Essa dor deveria compreender o período até sete dias antes do início da menstruação e/ou durante o período menstrual (iniciando em qualquer dia do período) e/ou ainda, iniciando imediatamente após o término da menstruação (até 24 horas após o término). A amostra selecionada devia apresentar os sintomas há pelo menos um ano e ter disponibilidade para participar do estudo. Em relação aos contraceptivos, foram aceitas as mulheres que não faziam uso e as que faziam também, desde que obrigatoriamente fossem contraceptivos orais e o intervalo mensal acontecesse para que as mulheres pudessem menstruar.

Como critérios de exclusão, tiveram-se: mulheres que já estavam na menopausa ou, por algum motivo, não estivessem menstruando; gestantes; mulheres que faziam uso de contraceptivos que não fossem os orais; mulheres que faziam uso de contraceptivos orais, mas não davam o intervalo entre as cartelas para que houvesse a menstruação; mulheres com menstruação desregular, ou seja, ciclo menor de 20 dias ou maior de 35 dias entre as menstruações; indivíduos que estivessem fazendo tratamento de acupuntura independentemente do motivo; indivíduos que haviam terminado um tratamento de acupuntura, independente também do motivo, há menos de seis meses; pacientes que estivessem fazendo outro tratamento paralelo para enxaqueca; indivíduos que faziam continuamente o uso de medicamentos com comprometimento sistêmico, tais como, medicamentos para hipertensão arterial, diabetes, antidepressivos, ansiolíticos, entre outros, exceto os contraceptivos.

Após divulgação do estudo e contato com os interessados, selecionou-se a amostra, que foi de 10 
voluntários. Esses indivíduos formaram um único grupo que foi chamado de Grupo Caso.

Para aplicação da técnica, foi realizada uma assepsia com álcool a $70 \%$ em todo o pavilhão auricular e o orifício da orelha foi protegido com algodão seco. A técnica de terapia auricular foi aplicada com sementes de mostarda auriculares, presas com fita micropore, em pontos eleitos descritos por Souza (2001): Shenmen, Simpático, Rim, Subcórtex, Endócrinas, Estômago e Encéfalo. A aplicação destes pontos foi feita de acordo com a descrição da medicina chinesa, ou seja, unilateralmente, na orelha direita para a mulher. Os pontos escolhidos são pontos que, segundo a medicina chinesa, tem indicação para a enxaqueca menstrual. As pacientes permaneceram com as sementes auriculares durante quatro dias, e foram treinadas logo na primeira sessão para a retirada das sementes auriculares; no oitavo dia a paciente voltava para receber nova aplicação em horário prédeterminado. $\mathrm{O}$ tratamento completo foi repetido durante doze sessões semanais consecutivas.

Para a avaliação da qualidade de vida, foi utilizado o questionário genérico Medical Outcomes Study Short-Form 36 (SF-36), cuja tradução e validação cultural no Brasil foi realizada por Ciconelli, Ferraz e Santos (1999), onde foram analisados os aspectos físicos e mentais. Este é um questionário amplamente utilizado na avaliação de qualidade de vida de pacientes com doenças crônicas. É composto por oito dimensões ou domínios: Capacidade Funcional (CF), Limitações por Aspectos Físicos (LAF), Dor Corporal (DC), Estado Geral de Saúde (EGS), Vitalidade (VT), Aspectos Sociais (AS), Aspectos Emocionais (AE) e Saúde Mental (SM). Essas escalas são pontuadas de 0 a 100 , e o maior valor indica melhor função e são apresentadas num Escore de Componente Físico (ECF) que inclui as dimensões CF, LAF, DC, EGS e VT; e num Escore de Componente Mental (ECM) que inclui as dimensões AS, AE, SM, EGS e VT. Apesar de ser um questionário auto-aplicável, optou-se por entrevista direta aplicada pela pesquisadora, para que o paciente não tivesse dúvidas no entendimento das questões. Este questionário foi aplicado em três etapas durante o estudo: 1) antes de iniciar o tratamento, imediatamente após a $1^{\mathrm{a}}$ avaliação do questionário de $\mathrm{QV}$, a paciente recebeu a $1^{\mathrm{a}}$ aplicação da terapia auricular; 2) sessenta dias após o início do tratamento e, por fim, 3) noventa dias após o início do tratamento.

Para a análise estatística deste estudo, optouse por uma "Análise de Crescimento de Número Índices", na qual se obteve um resultado em porcentagem da evolução dos indivíduos durante o período do tratamento observando o crescimento ou decréscimo nos três períodos de aplicação do SF36. Para isso, foram utilizadas as fórmulas:

Base móvel $\operatorname{NIF}_{\left(t_{0}, t_{1}\right)}=\left(\frac{X_{i}}{X_{b}} 100\right)-100$

onde: $\mathrm{t}_{0} \rightarrow$ é o tempo fixado, ano base; $\mathrm{t} 1 \rightarrow$ é o tempo final e a Taxa média de crescimento

$$
r=\frac{100 x\left(\frac{\Delta P_{0}}{P_{0}}\right)}{n}
$$

onde: $\mathrm{P}_{0} \rightarrow$ É a população inicial do período em estudo; $\mathrm{P}_{\mathrm{i}} \rightarrow$ É a população final do período em estudo; $\Delta \mathrm{P}_{0} \rightarrow$ É a diferença entre o período inicial e o final do período em estudo $\mathrm{P}_{\mathrm{i}}-\mathrm{P}_{0} ; \mathrm{n} \rightarrow$ é o número de anos decorridos no período em estudo.

\section{Resultados e discussão}

De acordo com os critérios de exclusão, duas pacientes estudadas foram excluídas do estudo, por motivo de desistência. Elas não completaram o tempo total de tratamento e, assim, não realizaram a avaliação final. Portanto, os resultados deste estudo foram obtidos em oito das dez pacientes previamente selecionadas.

A média de idade das oito participantes estudadas é de 25,5 $\pm 6,5$ anos, e o tempo médio que essas mulheres lidam com a migrânea é de $8,1 \pm 8,1$ anos. 
Primeiramente, serão apresentados os resultados de acordo com a evolução de cada sujeito analisado para cada domínio, apresentando a taxa média de crescimento durante o tratamento.

Após será apresentado para cada domínio a taxa de crescimento e decréscimo encontrados nas avaliações realizadas durante as três fases do tratamento, para cada sujeito. A fase I compreende a fase antes de iniciar o tratamento, a fase II indica 60 dias após o início do tratamento e a fase III indica 90 dias após início do tratamento. Também se estabelecerá relação do crescimento da fase I para a fase II, e da fase II para a fase III, com as discussões pertinentes.

Tabela 1. Resultado da taxa média de crescimento dos sujeitos pesquisados com relação aos domínios

\begin{tabular}{lccccccccccc}
\hline SUJEITO & CF & LAF & DC & EGS & VT & AS & AE & SM & ECF & ECM & SF-36 \\
\hline 1 & 2,63 & 0,00 & 0,00 & 3,48 & $-15,38$ & 8,00 & - & $-7,50$ & $-1,40$ & 14,67 & 8,81 \\
2 & 10,71 & 0,00 & 32,42 & 53,30 & 50,00 & 325,00 & 0,00 & $-2,94$ & 21,98 & 8,01 & 9,12 \\
3 & $-28,95$ & $-50,00$ & $-32,31$ & 2,88 & $-11,54$ & $-33,33$ & $-50,00$ & $-8,82$ & $-25,09$ & $-16,08$ & $-25,65$ \\
4 & 0,00 & 0,00 & 0,00 & 0,00 & 15,38 & 0,00 & 0,00 & 13,89 & 2,24 & 4,61 & 2,79 \\
5 & 5,88 & $-37,50$ & 66,21 & 41,77 & 30,00 & 0,00 & 0,00 & 5,26 & 4,86 & 10,66 & 3,35 \\
6 & 2,94 & 25,00 & 9,69 & 0,00 & 12,50 & $-43,33$ & 100,15 & 2,94 & 8,04 & 17,41 & 14,72 \\
7 & 26,92 & 50,00 & 9,69 & 21,32 & 31,25 & 0,00 & 25,08 & 3,33 & 26,94 & 14,21 & 19,24 \\
8 & 0,00 & 0,00 & 46,34 & 3,48 & 0,00 & $-14,37$ & 0,00 & $-7,50$ & 7,10 & $-4,11$ & 1,25 \\
\hline
\end{tabular}

\section{Em relação a cada sujeito analisado}

A Tabela 1 apresenta os resultados da taxa média de crescimento calculada a partir da fórmula apresentada na metodologia. Cabe salientar que a taxa média leva em conta o início e o final do período do tratamento e as três fases envolvidas.

Nota-se que o sujeito 1 apresentou taxa média de crescimento ( $r$ ) positiva em cinco dos onze domínios estudados, CF, EGS, AS, ECM e SF36, e o maior crescimento ocorreu no ECM com $r$ de 14,67\%. Quanto ao decréscimo, os mesmos ocorreram nos domínios, VT, SM, ECF, com maior decréscimo no VT com $r=-15,38 \%$. Nos demais domínios, têm-se uma média de crescimento estacionária em LAF e DC, ou seja, terminou o tratamento com a mesma pontuação inicial. Cabe salientar ainda que, para o domínio AE, o mesmo apresentou um ponto inicial de zero mostrando, portanto um crescimento ao longo do tratamento, mas inviabilizando o cálculo da taxa média de crescimento.

Com relação ao sujeito 2, este foi o que melhor respondeu ao tratamento apresentando apenas decréscimo médio de 2,94\% no domínio SM e uma média estacionária no domínio LAF e AE. Nota-se que em relação aos demais pacientes tratados, o sujeito 2 foi o que apresentou maior taxa média de crescimento chegando a obter como resultado um $r$ de 325,00\% no AS. Quanto ao LAF e AE, a taxa média foi estacionária já que este iniciou o tratamento com pontuação máxima de $100 \%$.

$\mathrm{Na}$ continuidade, o sujeito 3 , de acordo com os resultados apresentados, teve apenas uma taxa 
com crescimento positivo o qual ocorreu para o domínio EGS e com resultado muito pequeno, ou seja, com $r=2,88 \%$. Com relação aos decréscimos em dois domínios, perdeu em média 50,00\% das funções iniciais e isto ocorreu em LAF e AE.

Os resultados obtidos para o sujeito 4 mostraram que o mesmo não apresentou decréscimo, mas não obteve resposta alguma em seis dos domínios apresentados, CF, LAF, DC, EGS, AS e AE. A melhora mais evidente foi obtida para o domínio VT com taxa média de 15,38\%.

Os resultados da taxa média $r$ para o sujeito 5 mostram uma resposta satisfatória ao tratamento uma vez que a taxa média foi positiva para oito dos onze domínios sendo que a maior taxa foi obtida no domínio dor (DC) com $\mathrm{r}=66,21 \%$. A tabela 1 mostra ainda que este sujeito não apresentou nenhum decréscimo em nenhum domínio e uma taxa estacionária nos domínios AS e AE.

Quanto ao sujeito 6, este apresentou apenas um decréscimo no tratamento e isso ocorreu para o domínio AS com taxa de $-43,33 \%$ e um resultado estacionário no domínio EGS. A maior taxa de crescimento foi de $100,15 \%$ para o AE.
Com relação ao sujeito 7 , dos onze domínios apenas o domínio AS apresentou resultado estacionário. Nos demais domínios, todos os resultados foram positivos com a taxa média crescente, sendo que o mais marcante foi de $50,00 \%$ para o domínio LAF.

No último paciente pesquisado (sujeito 8 ), os resultados da taxa média foram muito variados, ou seja, dos onze domínios tem-se quatro resultados estacionários, quatro com taxa média positiva e três com taxa média negativa. O resultado mais marcante foi para dor (DC) com taxa média de crescimento de $46,34 \%$.

Com os resultados acima, percebe-se que cinco dos sujeitos pesquisados apresentaram em sua maioria resultados com taxas médias positivas, mostrando benefícios como resposta do tratamento. O sujeito 7 foi o que melhor respondeu ao tratamento apresentando apenas um domínio estacionário e os demais com resultados positivos. Já o sujeito 3 foi o que apresentou o pior resultado com apenas um domínio com taxa média positiva.

Tabela 2. Taxa de crescimento da capacidade funcional nas três fases do tratamento

\begin{tabular}{llllcc}
\hline SUJEITO & I & II & III & I para II & II para III \\
\hline 1 & 95 & 100 & 100 & 5,26 & 0,00 \\
2 & 70 & 90 & 85 & 28,57 & $-5,56$ \\
3 & 95 & 90 & 40 & $-5,26$ & $-55,56$ \\
4 & 100 & 100 & 100 & 0,00 & 0,00 \\
5 & 85 & 90 & 95 & 5,88 & 5,56 \\
6 & 85 & 90 & 90 & 5,88 & 0,00 \\
7 & 65 & 95 & 100 & 46,15 & 5,26 \\
8 & 100 & 100 & 100 & 0,00 & 0,00 \\
\hline
\end{tabular}




\section{Em relação a cada domínio analisado}

\section{Capacidade Funcional (CF)}

A Tabela 2 mostra que a CF da fase I para a fase II apresenta como resultado negativo no tratamento para apenas um caso com decréscimo de 5,26\%. Para os sujeitos com resultados positivos no tratamento, tem-se como melhor resultado um sujeito que atingiu $46,15 \%$ e o segundo melhor atingiu um índice de 28,57\%. Os demais tiveram uma média de melhora de $5,67 \%$ em três casos. Houve ainda dois sujeitos que iniciaram o tratamento com $100 \%$ das suas condições nesse domínio e assim se mantiveram com o tratamento.

Já na CF da fase II para a fase III, além dos dois sujeitos que iniciaram com a condição máxima, ocorreu a inclusão de mais um sujeito, que obteve o máximo de resultado com tratamento (sujeito 1). Cabe salientar que o mesmo já estava bem próximo da condição máxima e, com apenas 5,26\% de acréscimo, o mesmo já obteve o máximo de resultado com o tratamento. O problema desta fase na CF ocorreu com o sujeito 3, pois o mesmo já se encontrava com perda no tratamento e aumentou essá perda em 55,56\%. Outro sujeito que não apresentava perda da $1^{\mathrm{a}}$ para $2^{\mathrm{a}}$ fase, foi o sujeito 2 , que passou a ter uma perda de $5,56 \%$.

A capacidade funcional se refere à capacidade de o indivíduo realizar suas atividades rotineiras do dia-a-dia como higiene, alimentação e vestuário, de forma independente e com possibilidades físicas e mentais de realizá-las (ROSA et al., 2003; FRANK et al., 2007).

Um estudo realizado por Mendonça (2006) analisou a capacidade funcional de mulheres fibromiálgicas submetidas ao tratamento de acupuntura por três meses e avaliadas por um questionário de qualidade de vida específico para fibromiálgicos (Fibromyalgia Impact Questionnaire). Esse estudo não encontrou uma melhora satisfatória na capacidade funcional antes e após o período do tratamento, alegando que este quesito era muito subjetivo. Ao contrário do que foi encontrado nesta literatura, o presente estudo demonstra que a terapia auricular foi eficaz para aumentar a funcionalidade da maioria dos indivíduos para este domínio, mesmo que, para alguns, esse aumento seja sutil.

Tabela 3. Taxa de crescimento das limitações por aspectos físicos nas três fases do tratamento

\begin{tabular}{lccccc}
\hline SUJEITO & I & II & III & I para II & II para III \\
\hline 1 & 75,0 & 100,0 & 75,0 & 33,33 & $-25,00$ \\
2 & 100,0 & 100,0 & 100,0 & 0,00 & 0,00 \\
3 & 100,0 & 50,0 & 0,0 & $-50,00$ & $-100,00$ \\
4 & 100,0 & 100,0 & 100,0 & 0,00 & 0,00 \\
5 & 100,0 & 50,0 & 25,0 & $-50,00$ & $-50,00$ \\
6 & 50,0 & 75,0 & 75,0 & 50,00 & 0,00 \\
7 & 50,0 & 100,0 & 100,0 & 100,00 & 0,00 \\
8 & 100,0 & 100,0 & 100,0 & 0,00 & 0,00 \\
\hline
\end{tabular}




\section{Limitações por Aspectos Físicos (LAF)}

$\mathrm{Na}$ tabela 3, tem-se que os indivíduos 3 e 5 perderam suas funções por aspectos físicos ao longo do período de tratamento, e essa situação é marcante, dado que os dois iniciaram com $100 \%$ das habilidades. Nota-se que o sujeito 3 chegou a perder totalmente as funções, e atingiu um decréscimo de $100 \%$. O sujeito 1 atingiu 33,33\% de melhora na fase I para fase II e chegou ao nível máximo de melhor função, mas na continuidade perdeu $25,00 \%$, voltando às condições iniciais do tratamento.
Outro aspecto interessante mostrado na tabela 3 ocorre com o sujeito 5 , que perdeu $50,00 \%$ a cada fase, mas não chegou a zerar sua limitação neste domínio. O tratamento mostra ainda uma melhora máxima que ocorreu com o sujeito 7 , pois da $1^{\mathrm{a}}$ para $2^{\mathrm{a}}$ fase o mesmo cresceu $100 \% \mathrm{e}$ ainda se manteve assim até o final do tratamento.

Três sujeitos $(2,4,8)$ mantiveram $100 \%$ das funções para este domínio durante todo o período do tratamento, sendo que todos já iniciaram com $100 \%$ das habilidades.

Tabela 4. Taxa de crescimento da saúde mental nas três fases do tratamento

\begin{tabular}{llllcc}
\hline SUJEITO & I & II & III & I para II & II para III \\
\hline 1 & 80 & 76 & 68 & $-5,00$ & $-10,53$ \\
2 & 68 & 60 & 64 & $-11,76$ & 6,67 \\
3 & 68 & 60 & 56 & $-11,76$ & $-6,67$ \\
4 & 72 & 80 & 92 & 11,11 & 15,00 \\
5 & 76 & 84 & 84 & 10,53 & 0,00 \\
6 & 68 & 72 & 64 & 17,65 & $-10,00$ \\
7 & 60 & 72 & 68 & $-20,00$ & $-11,11$ \\
8 & 80 & 64 & & & 6,25 \\
\hline
\end{tabular}

\section{Saúde Mental (SM)}

Os resultados da taxa de crescimento, para o domínio da SM da $1^{\mathrm{a}}$ para $2^{\mathrm{a}}$ fase, mostram que $50,00 \%$ dos sujeitos apresentam decréscimo no tratamento e os outros 50,00\% melhora no tratamento. Na $2^{\mathrm{a}}$ para a $3^{\mathrm{a}}$ fase, aparecem $40,00 \%$ dos resultados com taxas negativas, um sujeito com efeito estacionário e três com taxas positivas, onde a mais marcante é o do sujeito 8, uma vez que o mesmo apresentava a maior taxa negativa na fase I para II.
De acordo com o Relatório Mundial da Saúde (WORLD HEALTH ORGANIZATION, 2009, p. 31-32):

[...] estudiosos de diferentes culturas definem diversamente a saúde mental. Os conceitos de saúde mental abrangem, entre outras coisas, o bem-estar subjectivo, a auto-eficácia percebida, a autonomia, a competência, a dependência intergeracional e a auto-realização do potencial intelectual e emocional da pessoa. Numa perspectiva transcultural, é quase impossível definir saúde mental de uma forma completa. De 
um modo geral, porém, concorda-se quanto ao facto de que a saúde mental é algo mais do que a ausência de perturbações mentais.

Apesar de o próprio questionário separar os domínios de LAF e SM em dois grandes componentes diferentes (juntamente com outros domínios), o Componente Físico e o Componente Mental, respectivamente, sabe-se que esses sistemas estão interligados e interferem indiretamente um no outro.

No estudo de Cordeiro (1994 apud SILVA, 2007), a origem do psíquico e do físico para a MTC é através da mesma essência. Assim, para a MTC a saúde de um indivíduo é resultado do equilíbrio entre todos os sistemas internos físicos e psíquicos. As desarmonias e desequilíbrios podem ser verificados por meio de sinais que levarão a um sofrimento físico ou mental. Assim, pode-se dizer que existe uma interação entre esses dois aspectos e que as atividades mentais influenciam na energia do físico e que o contrário também é verdadeiro.

Ao observar os decréscimos dos domínios LAF e SM, pode-se visualizar que essa interação entre físico e mental realmente acontece. O domínio $\mathrm{SM}$ teve um maior número de indivíduos com decréscimos das porcentagens, se comparado com LAF.

O tratamento da acupuntura busca a harmonia perdida, trazendo o equilíbrio entre as unidades psíquicas e energéticas (SILVA, 2007). Segundo os resultados apresentados, pode-se dizer que ainda estava ocorrendo um equilíbrio entre essas unidades, ou que esse equilíbrio foi atingido, o que explica a taxa de crescimento das porcentagens e manutenção da melhor função para os domínios LAF e SM encontrados em alguns indivíduos.

Tabela 5. Taxa de crescimento dos aspectos emocionais nas três fases do tratamento

\begin{tabular}{lccccc}
\hline SUJEITO & I & II & III & I para II & II para III \\
\hline 1 & 0 & 66,6 & 100 & -1 & 50,15 \\
2 & 100 & 33,3 & 100 & $-66,70$ & 200,30 \\
3 & 33,3 & 66,6 & 0 & 100,00 & $-100,00$ \\
4 & 100 & 100 & 100 & 0,00 & 0,00 \\
5 & 100 & 100 & 100 & 0,00 & 0,00 \\
6 & 100 & 100 & 200,30 & 0,00 \\
7 & 33,3 & 100 & 100 & 50,15 & 0,00 \\
8 & 66,6 & 100 & 100 & 0,00 & 0,00 \\
\hline
\end{tabular}

\section{Aspectos Emocionais (AE)}

Ao se analisar a Tabela 5, em primeiro lugar cabe salientar que o sujeito 1 inicia o tratamento sem condições no domínio quanto aos AE, pois apresenta nível inicial de zero, e isso gera dificuldade no cálculo da taxa de crescimento. Como não existe ponto de partida para se avaliar o "crescimento", neste caso qualquer resultado pode ser visto como $100,00 \%$ de melhora ou, mesmo, $66,6 \%$ de melhora. Para os demais resultados notase que no domínio referente aos $\mathrm{AE}$ da $1^{\mathrm{a}}$ para a 
$2^{\mathrm{a}}$ fase, 3 sujeitos obtiveram taxa de crescimento positiva, mostrando melhora no tratamento. $\mathrm{O}$ maior aumento ocorreu com o sujeito 6 o qual melhorou em 200,30\% em relação à chegada para o tratamento. Nessa fase, ocorre ainda o fato de que quatro sujeitos $(2,4,5,8)$ já iniciaram o tratamento com o máximo das habilidades nos AE. Da $2^{\mathrm{a}}$ para $3^{\text {a }}$ fase, o sujeito que apresentou o melhor resultado foi o 2 que passou de uma taxa negativa de $66,70 \%$ para uma positiva de $200,30 \%$.

De uma maneira geral, com relação aos $\mathrm{AE}$ houve uma melhora com o tratamento. Um resultado que se mostra atípico é o caso do sujeito 3 , pois o mesmo melhorou em $100,00 \%$ na passagem da fase I para a fase II do tratamento, e na evolução da fase II para a fase III, perdeu 100,00\%.

Para a MTC, as emoções são estímulos mentais que influenciam a nossa vida afetiva e, em condições normais, não causam doenças. No Ocidente acredita -se que a fisiologia emocional e os processos mentais são atribuídos ao cérebro, porém a MTC acredita que eles são parte de ações dos sistemas internos. Os órgãos internos constituem uma energia mental própria positiva, essa energia só vai se tornar negativa quando for provocada por circunstâncias externas, por isso o estado dos órgãos internos afeta nosso estado emocional (MACIOCIA et al., 1996).

A grande resposta positiva encontrada neste domínio por meio da terapia auricular pode ser o fato de o tratamento ter incluindo o ponto Rim, órgão responsável pelas emoções de medo, trauma e culpa (MACIOCIA et al., 1996). Frequentemente, quando se comenta com pacientes enxaquecosos qual a emoção que está relacionada com a crise, estes referem o medo. A dor muitas vezes é tão forte que remete uma sensação de morte, causando dessa forma o medo, o pânico.

Tabela 6. Taxa de crescimento da dor corporal nas três fases do tratamento

\begin{tabular}{lccccc}
\hline SUJEITO & I & II & III & I para II & II para III \\
\hline 1 & 50,9 & 61,0 & 50,9 & 19,84 & $-16,56$ \\
2 & 50,9 & 83,9 & 83,9 & 64,83 & 0,00 \\
3 & 61,9 & 21,9 & 19,39 & $-70,37$ \\
4 & 83,9 & 83,9 & 83,9 & 0,00 & 0,00 \\
5 & 21,9 & 40,9 & 50,9 & 86,76 & 24,45 \\
6 & 61,9 & 73,9 & 73,9 & 19,39 & 0,00 \\
7 & 61,9 & 100,0 & 73,9 & 61,55 & $-26,10$ \\
8 & 51,9 & 100,0 & 100,0 & 92,68 & 0,00 \\
\hline
\end{tabular}

\section{Dor corporal (DC)}

Com relação ao domínio $\mathrm{DC}$, a tabela 6 mostra que da $1^{\mathrm{a}}$ para a $2^{\mathrm{a}}$ fase quase todos os sujeitos mostram diminuição da dor representada pelas taxas de crescimento positivas. Somente o sujeito 4 apresentou um resultado estacionário, mas, em relação aos demais, foi o que iniciou o tratamento com o resultado mais relevante de $83,9 \%$. Os resultados da $2^{\mathrm{a}}$ para a $3^{\mathrm{a}}$ fase mostram três sujeitos 
com resultados de taxa negativa e quatro que estacionaram no tratamento.

De acordo com a literatura, pela dor ser um caráter subjetivo, e como o indivíduo é singular, espera-se que cada um responda de forma diferente às intervenções externas, da mesma forma que não se pode pretender que mantenham uniformemente suas respostas a longo prazo (MICELI, 2002). Assim, pode-se entender melhor o porquê de alguns indivíduos evoluírem mais que outros na melhora da DC, ou até mesmo piorar após algum tempo mesmo com o tratamento, como mostrado neste estudo, pois cada um interpretará sua dor de forma particular. Ainda é comum relatos de pacientes nos consultórios, falando que já não lembram mais da dor inicial, e que o parâmetro que usam para graduar a dor é o atual, para as pesquisas isso influencia negativamente.

Para a MTC, a dor é causada por uma obstrução do fluxo da energia vital no corpo do indivíduo, e pode ser causada por fatores internos, externos climáticos e outros (WINK; CARTANA, 2007). Assim, a diminuição ou eliminação do quadro doloroso não depende somente do tratamento da dor em si, mas também dos sistemas que com ele interagem, como também as possibilidades de sua superação.

Vários estudos têm pesquisado a eficácia da acupuntura no tratamento da dor. Um estudo realizado por Usichenko et al. (2007) utilizou a terapia auricular para reduzir a dor pós-operatória da artroscopia de joelho, e observou que pacientes que receberam a aplicação dos pontos auriculares Shenmen, Pulmão e Articulação do Joelho, necessitaram de doses menores do medicamento ibuprofeno para alívio da dor, quando comparados com o grupo controle que também recebeu a terapia auricular, mas não para analgesia.

Tabela 7. Taxa de crescimento da vitalidade nas três fases do tratamento

\begin{tabular}{lllccc}
\hline SUJEITO & I & II & III & I para II & II para III \\
\hline 1 & 65 & 55 & 45 & $-15,38$ & $-18,18$ \\
2 & 35 & 65 & 70 & 85,71 & 7,69 \\
3 & 65 & 65 & 50 & 0,00 & $-23,08$ \\
4 & 65 & 80 & 85 & 23,08 & 6,25 \\
5 & 50 & 80 & 70,00 & $-5,88$ \\
6 & 40 & 55 & 50 & 62,50 & $-23,08$ \\
7 & 40 & 55 & 65 & 37,50 & 18,18 \\
8 & 50 & 40 & 50 & $-20,00$ & 25,00
\end{tabular}

\section{Vitalidade (VT)}

A Tabela 7 apresenta para o domínio VT da $1^{\mathrm{a}}$ fase para a $2^{\mathrm{a}}$ fase, dois resultados com taxa negativa, o sujeito 1 com $15,38 \%$ e o sujeito 8 com $20,00 \%$; mostra ainda o sujeito 3 sem reação ao tratamento. $\mathrm{O}$ resultado positivo mais expressivo foi de $85,71 \%$ do sujeito 2. Da $2^{\mathrm{a}}$ para a $3^{\mathrm{a}}$ fase, o número de resultados negativos aumentou, aparecendo em 
quatro sujeitos.

Maciocia et al. (1996) fala das substâncias vitais, que são o Qi (Energia), Sangue (Xue), Essência (Jing) e Fluídos Corpóreos (Jin Ye). As principais funções dos sistemas internos são de assegurar que as substâncias vitais sejam mantidas, abastecidas, transformadas e movimentadas de forma correta no nosso organismo, logo isso nos dá a vitalidade, segundo a MTC.

Tabela 8. Taxa de crescimento dos aspectos sociais nas três fases do tratamento

\begin{tabular}{lccccc}
\hline SUJEITO & I & II & III & I para II & II para III \\
\hline 1 & 75 & 87 & 87 & 16,00 & 0,00 \\
2 & 10 & 50 & 75 & 400,00 & 50,00 \\
3 & 75 & 62 & 25 & $-17,33$ & $-59,68$ \\
4 & 10 & 10 & 10 & 0,00 & 0,00 \\
5 & 75 & 75 & 0,00 & 0,00 \\
6 & 75 & 10 & 10 & $-86,67$ & 0,00 \\
7 & 75 & 75 & 0,00 & 0,00 \\
8 & 87 & 62 & 62 & $-28,74$ & 0,00 \\
\hline
\end{tabular}

\section{Aspectos Sociais (AS)}

A evolução do tratamento apresentada pela Tabela 8 com relação à taxa de crescimento para o domínio dos AS mostra um aumento marcante para o sujeito 2 da $1^{\mathrm{a}}$ para a $2^{\mathrm{a}}$ fase, o qual evoluiu em 400,00\% em relação ao início do tratamento. Houve, ainda nessa fase, três sujeitos $(4,5,7)$ com valores estacionários e três com decréscimos $(3,6,8)$. Da $2^{\mathrm{a}}$ para a $3^{\mathrm{a}}$ fase, a maioria dos sujeitos estacionou no tratamento. Nessa fase, o resultado mais marcante foi para o sujeito 2 o qual aumentou em 400,00\% em um primeiro momento e aumentou mais $50,00 \%$ no segundo momento.

Tanto o sujeito 2 como o sujeito 4 , iniciaram o tratamento com uma baixíssima porcentagem de função para o domínio $\mathrm{AS}$, mas somente o indivíduo 2 conseguiu evoluir durante $\mathrm{o}$ tratamento otimizando os resultados, e o sujeito 4 não teve respostas de melhora e nem piora para este domínio. É muito provável que a terapia auricular para o sujeito 2 conseguiu de alguma forma fazer com que esse indivíduo melhorasse sua vida social e foi quem apresentou a maior taxa média de crescimento. O contrário ocorreu com o sujeito 4, que, de forma geral, apresentou resultados estacionários em grande parte dos domínios analisados.

Conforme relata a literatura, ao tratar um paciente além de considerar os fatores biológicos e psicológicos desencadeantes das patologias, deve-se considerar também as condições sociais, familiares e econômicas. Não basta tratar somente a dor, mas também o paciente, e além dele, os sistemas que com ele interagem (MICELI, 2002). 
Tabela 9. Taxa de crescimento do estado geral de saúde nas três fases de tratamento

\begin{tabular}{lccccc}
\hline SUJEITO & I & II & III & I para II & II para III \\
\hline 1 & 71,9 & 76,9 & 76,9 & 6,95 & 0,00 \\
2 & 46,9 & 91,9 & 96,9 & 95,95 & 5,44 \\
3 & 86,9 & 91,9 & 91,9 & 5,75 & 0,00 \\
4 & 96,9 & 91,9 & 96,9 & $-5,16$ & 5,44 \\
5 & 41,9 & 66,9 & 76,9 & 59,67 & 14,95 \\
6 & 86,9 & 86,9 & 06,9 & 0,00 & 0,00 \\
7 & 46,9 & 66,9 & 66,9 & 42,64 & 0,00 \\
8 & 71,9 & 81,9 & 76,9 & 13,91 & $-6,11$ \\
\hline
\end{tabular}

\section{Estado geral de Saúde (EGS)}

De acordo com a taxa de crescimento apresentada pela tabela 9 , os resultados da $1^{\mathrm{a}}$ para a $2^{\mathrm{a}}$ fase, mostram apenas um resultado negativo na taxa de crescimento obtida pelo sujeito 4 com decréscimo de $5,16 \%$. O maior efeito positivo no tratamento ocorreu para o sujeito 2 com $95,95 \%$ de melhora, seguido pelo sujeito 5 com $59,67 \%$ e pelo sujeito 7 com $42,64 \%$, e estes três sujeitos, antes de iniciar o tratamento, estavam abaixo dos $50,00 \%$ indicando uma função ruim para este domínio. O sujeito 6 não obteve qualquer reação ao tratamento. Já da $2^{\mathrm{a}}$ para a $3^{\mathrm{a}}$ fase o sujeito 8 decaiu com relação à fase anterior para $6,11 \%$; três dos sujeitos $(2,4,5)$ aumentaram a taxa de crescimento e quatro $(1,3,6$, 7) estacionaram.

O EGS está inserido tanto no Componente Mental quanto no Componente Físico, ou seja, é a saúde como um todo do indivíduo. Como foi observado nos resultados dos outros domínios, houve boas melhoras da função destes durante o tratamento, assim, já se esperava uma melhora deste domínio. Portanto, o tratamento pela terapia auricular neste estudo foi eficaz para proporcionar uma melhora da função do Estado Geral de Saúde nos indivíduos analisados.

Tabela 10. Taxa média de crescimento do SF-36 nas três fases do tratamento

\begin{tabular}{lccccc}
\hline SUJEITO & I & II & III & I para II & II para III \\
\hline 1 & 64,1 & 77,8 & 75,4 & 21,37 & $-3,08$ \\
2 & 71,3 & 71,7 & 84,3 & 0,56 & 17,57 \\
3 & 73,1 & 70 & 35,6 & $-4,24$ & $-49,14$ \\
4 & 89,7 & 91,9 & 94,7 & 2,45 & 3,05 \\
5 & 68,7 & 73,9 & 73,3 & 7,57 & $-0,81$ \\
6 & 62,5 & 83,8 & 80,9 & 34,08 & $-3,46$ \\
7 & 58,2 & 82,9 & 80,6 & 42,44 & $-2,77$ \\
8 & 80,1 & 81 & 82,1 & 1,12 & 1,36 \\
\hline
\end{tabular}




\section{Qualidade de Vida Geral (SF-36)}

De acordo com os resultados obtidos na tabela 10 a melhora no tratamento com relação ao SF-36, ocorreu de forma mais marcante da $1^{\mathrm{a}}$ para a $2^{\mathrm{a}}$ fase, quando apenas o sujeito 3 apresentou decréscimo na taxa média de 4,24\%. Os sujeitos 6 e 7 foram os que obtiveram as maiores taxas com $34,08 \%$ e $42,44 \%$. Já da $2^{\mathrm{a}}$ para a $3^{\mathrm{a}}$ fase os resultados mostram aumento de sujeitos com taxas médias negativas, dos quais a mais marcante foi para o sujeito 3 com $49,14 \%$.

De forma geral, teve-se melhora na qualidade de vida dos pacientes estudados. Obtiveram melhora, mesmo aqueles que da $2^{\mathrm{a}}$ para a $3^{\mathrm{a}}$ fase apresentaram decréscimo, quando comparados com o início do tratamento (fase I). Assim, dos oito indivíduos estudados, sete tiveram uma melhora geral da sua qualidade de vida.

A Organização Mundial da Saúde define qualidade de vida como "a percepção do indivíduo de sua posição na vida no contexto da cultura e sistema de valores nos quais ele vive e em relação aos seus objetivos, expectativas, padrões e preocupações". A qualidade de vida é influenciada de forma complexa pela saúde física do indivíduo, estado psicológico, nível de independência, relações sociais, crenças pessoais e as suas relações com aspectos do ambiente em que vive (FLECK et al., 1999, p. 20). Avaliar a qualidade de vida é uma tentativa de quantificar cientificamente as conseqüências do quanto uma doença e o seu tratamento afetam a habilidade de viver a vida de forma satisfatória sobre a percepção do paciente (SOUZA; PAIVA; REIMÃO, 2006).

\section{Considerações Finais}

Através do estudo realizado, concluiu-se que a terapia auricular melhorou a qualidade de vida da maioria dos indivíduos pesquisados, mulheres com migrânea menstrual.

Em relação a cada sujeito, percebeu-se que o sujeito 7 obteve os melhores resultados, pois apenas nos Aspectos Sociais manteve a taxa estacionária, e também não apresentou nenhum decréscimo nas suas funções durante o tratamento.

Já o sujeito 3 apresentou na maioria dos seus domínios decréscimo das funções. Vale salientar que o tratamento proposto baseou-se na aplicação de pontos fixos auriculares, indicados segundo a MTC para migrânea menstrual. Há pacientes, no entanto, que necessitariam de um acompanhamento mais individualizado, com diagnóstico próprio e uso de pontos específicos conforme suas alterações, talvez se este paciente fosse acompanhado dessa forma os resultados seriam também mais animadores.

Quando analisados os domínios na taxa média de crescimento dos sujeitos, de uma maneira geral, é no Estado Geral de Saúde, que se apresentam os melhores resultados, e nenhum paciente apresentou funções em decréscimo. Na sequência, temos a Capacidade Funcional e a Dor Corporal também com bons resultados. Esse dado é importante para o estudo, pois, quando se tem migrânea um dos principais anseios quando um paciente procura por tratamento é principalmente a melhora da sua dor e que mantenha suas capacidades, já que conforme dito na literatura, a migrânea muitas vezes é incapacitante, diminuindo o rendimento do paciente acometido.

Por fim, o estudo mostrou-se bastante recompensador, mostrando que é possível melhorar a qualidade de vida de mulheres com migrânea menstrual através da terapia auricular. Melhorar a qualidade de vida não significa somente melhorar a dor, mas sim intervir positivamente nas condições de vida, sejam elas físicas, sociais, emocionais e mentais.

\section{Referências}

ARAÚJO, A. P. S.; ZAMPAR, R.; PINTO, S. M. E. Auriculoterapia no tratamento de indivíduos acometidos por distúrbios osteomusculares relacionados ao trabalho (DORT) / lesões por esforços repetitivos (LER). Arquivo Ciência da Saúde Unipar, Umuarama, v. 10, n. 1, p. 3542, 2006. 
BIGAL, M. E.; FERNANDES, L. C.; MORAES, F. A.; BORDINI, C. A.; SPECIALI, J. G. Prevalência e impacto da migrânea em funcionários do hospital das clínicas da faculdade de medicina de Ribeirão Preto-USP. Arquivo de Neuro-psiquiatria, São Paulo, v. 58, n. 2-B, p. 431436, 2000.

CICONELLI, R. M.; FERRAZ, M. B.; SANTOS, W. Tradução para a língua portuguesa e validação do questionário genérico de avaliação de qualidade de vida SF-36 (Brasil SF-36). Revista Brasileira de Reumatologia, São Paulo, v. 39, n. 3, p. 143-150, 1999.

FLECK, M. P. A.; LEAL, O. F.; LOUZADA, S.; XAVIER, M.; CHACHAMOVICH, E.; SANTOS, G. L.; PINZON, V. Desenvolvimento da versão em português do instrumento de avaliação de qualidade de vida da OMS (WHOQOL-100). Revista Brasileira de Psiquiatria, São Paulo, v. 21, n. 1, p. 19-28, 1999.

FRANK, S.; SANTOS, S. M. A.; ASSMANN, A.; ALVES, K. L.; FERREIRA, N. Avaliação da capacidade funcional: repensando a assistência ao idoso na saúde comunitária. Estudos interdisciplinares sobre o envelhecimento, Porto Alegre, v. 11, p. 123-134, 2007.

GIONA, P. Abordagem fisioterapêutica nas cefaléias tensionais através da terapia manual: série de casos. 2003. Monografia (Graduação em Fisioterapia) Universidade Estadual do Oeste do Paraná, Cascavel.

GONZALES, E. G. Auriculoterapia: escola de Huang Li Chun. São Paulo: Roca, 2004.

KRYMCHANTOWSKY, A. V.; TAVERES, C.; PENTEADO, J. C.; ADRIANO, M. Topiramato no tratamento preventivo da migrânea: experiência em um certo terciário. Arquivo de Neuropsiquiatria, São Paulo, v. 62, n. 1, p. 91-115, 2004.

MACIOCIA, G.; YING, Z. Z.; LEITE, T. C.; LEITE, N. M. A prática da medicina chinesa: tratamento de doenças com acupuntura e ervas chinesas. São Paulo: Roca, 1996.

MENDONÇA, C. M. Eficácia da acupuntura sobre a dor e a qualidade de vida em mulheres com fibromialgia. Revista PIBIC, Osasco, v. 3, n. 1, p. 75-81, 2006.

MICELI, A. V. P. Dor crônica e subjetividade em oncologia. Revista Brasileira de Cancerologia, Rio de Janeiro, v. 48, n. 3, p. 363-373, 2002.

ROSA, T. E. C.; BENÍCIO, M. H. A.; LATORRE, M. R. D. O.; RAMOS, L. R. Fatores determinantes da capacidade funcional entre idosos. Revista Saúde Pública, São Paulo, v. 37, n. 1, p. 40-48, 2003.

SCOGNAMILLO-SZABÓ, M. V. R. S.; BECHARA, G. H. Acupuntura: bases científicas e aplicações. Revisão Bibliográfica. Ciência Rural, Santa Maria, v. 31, n. 6, p.
1091-1099, 2001.

SILVA, D. F. Psicologia e acupuntura: aspectos históricos políticos e teóricos. Psicologia Ciência e Profissão, Brasília, v. 27, n. 3, p. 418-129, 2007.

SOCIEDADE BRASILEIRA DE CEFALÉIA. Dor de cabeça: cefaléia na mulher. Disponível em: $<\mathrm{http}: / /$ www.sbce.med.br/dor_cabeca/enxaqueca_mulher.asp $>$. Acesso em: 24 jan. 2009.

SOUZA, J. A. Recomendações para o tratamento profilático da migrânea: consenso da Sociedade Brasileira de Cefaléia. Arquivo de Neuropsiquiatria, São Paulo, v. 6, n. 1, p. 159-169, 2000.

SOUZA, J. C.; PAIVA, T.; REIMÃO, R. Qualidade de vida de caminhoneiros. Jornal Brasileiro de Psiquiatria, Rio de Janeiro, v. 55, n. 3, p. 184-189, 2006.

SOUZA, M. P. Tratado de auriculoterapia. Brasília: Novo Horizonte, 2001.

USICHENKO, I. T.; KUCHLING, S.; WITSTRUCK, T.; PAVLOVIC, D.; ZACH, M.; HOFER, A.; MERK, H.; LEHMANN, C.; WENDT, M. Auricular acupuncture for pain relief after ambulatory knee surgery: a randomized trial. Canadian Medical Association or its licensors CMAJ, Ottawa, v. 176, n. 2, p. 179-183, 2007.

WINK, S.; CARTANA, M. H. F. Promovendo o autocuidado a pacientes com cefaléia por meio da perspectiva oriental de saúde. Revista Brasileira de Enfermagem, Rio de Janeiro, v. 60, n. 2, p. 225-228, 2007.

WORLD HEALTH ORGANIZATION. A saúde mental pelo prisma da saúde pública. Disponível em: $<\mathrm{http}: / /$ www.who.int/whr/2001/en/whr01_ch1_po.pdf $>$. Acesso em: 30 jun 2009.

ZUKERMAN, E. Enxaqueca. In: MELO-SOUZA, S. E. Tratamento das doenças neurológicas. Rio de Janeiro: Guanabara Koogan, 2000. p. 485-488.

Recebido em 23 de março de 2010 - Received on March 23, 2010

Aceito em 27 de outubro de 2010 - Accepted on October 27, 2010 
